\title{
BM) Open Quality Development and implementation of a structured ward round in acute adult psychiatry
}

\author{
Anna Rebecca Mattinson, ${ }^{1}$ Sarah Jane Cheeseman ${ }^{2}$
}

To cite: Mattinson AR, Cheeseman SJ. Development and implementation of a structured ward round in acute adult psychiatry.BMJ Open Quality 2018;7:e000035. doi:10.1136/ bmjoq-2017-000035

- Additional material is published online only. To view please visit the journal online (http://dx.doi.org/10.1136/ bmjoq-2017-000035).

Received 5 April 2017 Revised 1 June 2018 Accepted 14 June 2018
Check for updates

(C) Author(s) (or their employer(s)) 2018. Re-use permitted under CC BY-NC. No commercial re-use. See rights and permissions. Published by BMJ.

${ }^{1}$ National Education for Scotland GP Trainees, NHS Lothian,

Edinburgh, UK

${ }^{2}$ Department of Psychiatry, Royal Edinburgh Hospital, Edinburgh, UK

Correspondence to Dr Sarah Jane Cheeseman; jane.cheeseman@nhslothian. scot.nhs.uk

\section{ABSTRACT}

Delivering high quality care in acute psychiatry requires a coordinated approach from a multidisciplinary team (MDT). Weekly ward rounds are an important forum for reviewing a patient's progress and developing a personalised care plan for the coming week. In general medicine, structured ward rounds and check lists have been shown to prevent omissions and improve patient safety; however, they are not widely used in psychiatry. At the Royal Edinburgh Hospital, the format of ward rounds differed between psychiatry wards and clinical teams, and care plans were not standardised. An audit in October 2015 found only 5\% of acute psychiatric inpatients had a documented nursing care plan. It was agreed that a clear multidisciplinary care plan from the weekly ward round would be beneficial. A group of consultant psychiatrists identified seven key domains for ward round (Social needs, Community Mental Health Team liaison, Assessments required, Mental Health Act, Prescriptions: medication electroconvulsive therapy (ECT), T2/T3, Engagement with relatives and carers, Risk Assessment and Pass Plans). This was given the acronym SCAMPER. Following this, a clinical MDT on a paired male and female ward, developed and introduced a structured ward round sheet. Within 8 weeks this was being used for $100 \%$ of patients. It was subsequently introduced into three other acute adult psychiatry wards and the intensive psychiatric care unit. Staff feedback was sought verbally and via a questionnaire. This was positive. The form was widely accepted and staff felt it improved patient care and ward round quality.

\section{PROBLEM}

The Royal Edinburgh Hospital (REH) is the largest psychiatric hospital in Lothian, providing a full range of psychiatric services to 620000 people in the City of Edinburgh, East Lothian and Midlothian regions of Scotland. Acute adult psychiatry provides inpatient care to all adults aged 18-65 years and is spread across five wards and an intensive psychiatric care unit (IPCU).

Weekly ward rounds on the acute adult psychiatry wards varied in structure and content according to the ward and consultant. There was no standardised documentation system to support the ward round process and often weekly action plans, including clinical tasks to be undertaken by different team members, would not be clearly identified and documented. Indeed, an audit of all case notes on the acute adult psychiatry wards, carried out by an external consultancy firm, identified only $5 \%$ of patients to have a nursing care plan. It was agreed that a clear plan generated by the multidisciplinary team (MDT) (outlined in box 1) at weekly ward rounds would aid in the development of these. There was concern among medical and nursing staff that important clinical tasks (eg, referring to social work, updating families and cognitive assessments) were not being clearly communicated within the team. It was acknowledged that this was negatively impacting patient care by leading to omissions and delays, for example in making referrals, and possibly increasing length of stay for some patients.

The aim of the project was to develop, test and implement a tool to enable structured and thorough review of care at weekly ward rounds. The key goal was for all patients to have a structured and documented multidisciplinary review completed each week.

\section{BACKGROUND}

The Royal College of Psychiatrists sets standards that 'a full multidisciplinary review/ ward round occurs at least once a week' and that 'managers and practitioners have agreed standards for reviews/ward rounds'. In addition, reviews should facilitate carers' views to be expressed. ${ }^{1}$

Delivering high-quality care in acute psychiatry requires a coordinated approach from a MDT. Ward rounds allow for a timely discussion of a patients' diagnosis, progress, the formulation of an effective care plan and safe and efficient clinical handover within the team. The patient's risk assessment should be reviewed along with pass plans. The ward round should be holistic and structured to ensure that progress continues to be made, for example with social issues. This 


\section{Box 1 Multidisciplinary team in psychiatry}

Consultant psychiatrist.

Doctors in training.

Mental health nurses.

Community psychiatric nurses.

$>$ Social worker.

$\checkmark$ Pharmacist.

0ccupational therapist.

Medical and nursing students.

is essential to maintain patient safety and good clinical care. $^{2}$

In general medicine, structured ward rounds and check lists have been shown to prevent omissions in care and to improve patient safety. Acronyms and mnemonics are frequently used to improve recall of important information and tasks by the clinical team. ${ }^{3}$ In the acute wards of $\mathrm{REH}$, ward rounds did involve a MDT and reviewed care within a biopsychosocial model but there was no consistent and standardised means of documenting a structured review and action plan. A structured ward round had not previously been trialled at REH and a nationally recommended, evidence-based, structured ward round, does not exist in psychiatry nor have the authors been able to find examples from other countries.

\section{BASELINE MEASUREMENT}

An audit of acute adult psychiatry wards at REH in October 2015 , conducted by an external consultancy firm, identified only $5 \%$ of patients to have a nursing care plan. Verbal feedback from medical and nursing staff indicated frequent problems in communication and completion of clinical tasks. This, for example, could result in a delay in referring to social work, failure to complete cognitive assessments prior to the next ward round or forgetting to update a family. Consultants commented that, despite a biopsychosocial review of care during ward rounds, documentation of this and completion of tasks was often inconsistent. The impression was that this was leading to delays in patient care and progress towards discharge. It was decided by the clinical team on one acute adult psychiatry ward (Balcarres ward) that improvements in the quality and structure of ward rounds were needed to address this.

\section{DESIGN}

Seven key domains for a thorough ward round discussion and care plan were identified at a stakeholder workshop, attended by acute general adult psychiatrists. These were: Social needs, Community Mental Health Team liaison, Assessments required, Mental Health Act review, Prescriptions: medication, electroconvulsive therapy (ECT), T2/ T3 (Consent to Treatment forms), Engagement with carers and relatives, Risk assessment and review of pass plans. This was given the acronym SCAMPER. These

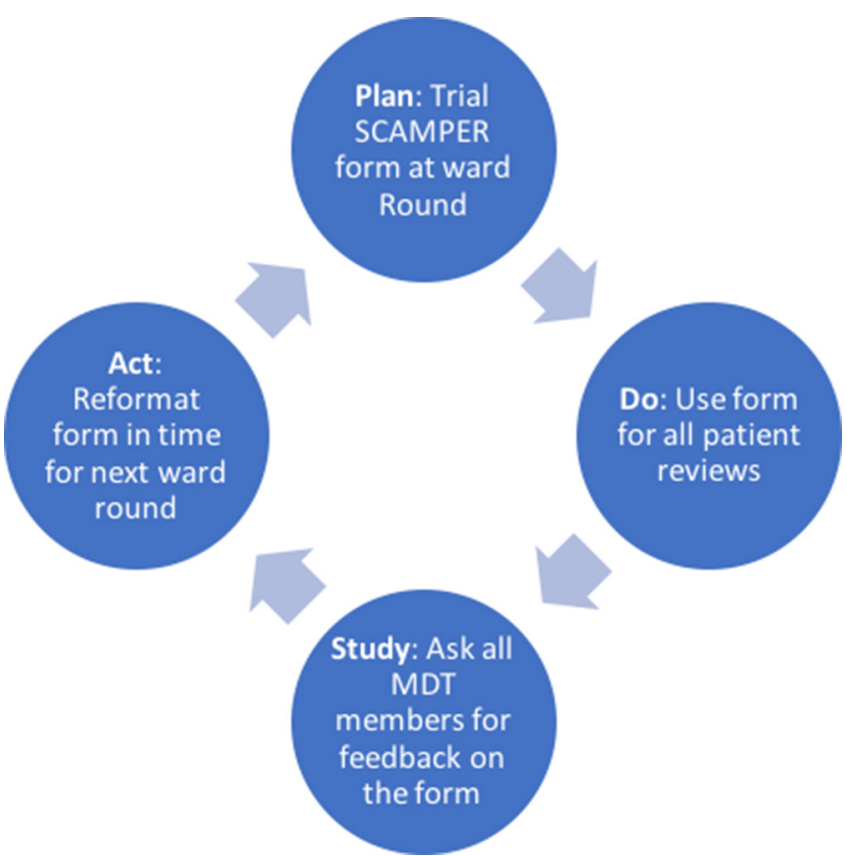

Figure 1 Example of PDSA cycle to improve SCAMPER ward round document. MDT, multidisciplinary team; PDSA, plan, do, study, act.

domains were chosen as they were pertinent to the care of every patient across diagnostic categories.

A similar version was devised for the IPCU, given the acronym SHAMPER. 'Community mental health team liaison' was replaced by 'Host ward liaison' as all patients are transferred back to their general adult host ward as a transition towards discharge. As such, this domain ensures there is effective communication of clinical progress between the inpatient teams.

A standardised ward round document (see online supplementary file 1) was devised by a project team on one paired male and female ward (Balcarres wards) around these seven domains. Sections for weekly nursing and medical staff review were included. This was first introduced in November 2015.

Plan, do, study, act (PDSA) cycles were carried out on a weekly basis by one consultant psychiatrist on the pilot wards, with support from the clinical team, to improve the format of the ward round document (see figure 1). Changes made during this process included adding a section for patient and carer views and requests, adding in an estimated discharge date and altering formatting of the document to make it more user friendly. In total, seven versions of the form were developed before a final document was agreed on. A 'Guide to SCAMPER' was designed (see online supplementary appendix 1) and distributed to all wards. This provided guidance on the use, completion and storage of the forms. Verbal information and support was also available. Compliance with the standardised ward round document was audited.

An initial questionnaire to assess awareness of SCAMPER, satisfaction and areas for improvement was conducted on the pilot wards in February 2016 (see 
online supplementary appendix 2). Feedback was positive and constructive.

The SCAMPER ward round document was subsequently introduced onto three other acute adult wards and the modified SHAMPER document into IPCU. By March 2016, all the acute adult wards and the IPCU were using the standardised ward round document. Again, compliance was audited and verbal feedback from staff was sought.

In May 2016, all staff members involved in patient care on the wards using SCAMPER/SHAMPER were invited to complete a questionnaire to obtain feedback on the standardised ward round document (see online supplementary appendix 3). Questionnaires were reviewed independently by both authors and recurrent themes were identified.

\section{STRATEGY}

The aim was to ensure that all patients in the acute general adult wards had a structured review of care, using the SCAMPER domains, completed at a weekly ward round by the MDT. The aim was to implement the new ward round tool in all acute adult wards by June 2016 . The underlying assumption was that implementation of this system redesign would lead to improvements in the reliable allocation and completion of necessary clinical tasks and a clearly documented risk assessment and management plan for all patients.

The initial phase was to develop a form to document a structured care plan that was designed around the use of the SCAMPER acronym. A SCAMPER form (see online supplementary file 1) was introduced and used with one consultant (author SJC) team at the weekly ward round. A balancing measure of the length of the ward round with the new form was incorporated.

A PDSA cycle was conducted weekly with this multidisciplinary ward team. Following each ward round verbal feedback on the clarity and format of the document was sought. Adequacy of space for each section and whether all aspects of care could be covered under the headings was discussed. Possible adaptations to the form were discussed and a new form was prepared for the following week's ward round (see figure 1). Changes included: combining sections physical and mental health assessments for a holistic overview of the patient's health and introducing a section specifically for prescriptions, including ECT. Space for a patient's and carer's requests and views was added as well as an estimated discharge date. No changes to the document impacted negatively on the ward round or had to be reversed. Many of the later PDSA cycles were focused on formatting the SCAMPER document to make it clearer and more user-friendly. After seven such PDSA cycles, a standardised form was agreed based on these experiences and team consensus.

The aim of the PDSA cycles was to ensure that the final form succinctly covered key aspects of care. It was recognised that an overly detailed form could lead to staff disengagement and, if ward rounds became inefficient, an unwillingness to use the tool. Balancing measures considered were: the preparation time for nurses to complete the review section as well as the length of the ward round.

The second phase was to introduce the form to all consultant teams on the Balcarres male and female wards. Its use was monitored to demonstrate that the approach could be successfully implemented.

Once a reliable process was embedded on the Balcarres wards, a meeting was held in December 2015 with two more general adult wards and the IPCU to discuss how to approach the spread of the new ward round documentation system to other wards. Representatives from the MDTs attended. The forms were subsequently introduced onto these wards. The completion of the SCAMPER domains were audited on each of the five wards, starting on IPCU $(8 / 2 / 2016)$, Meadows male and female wards $(1 / 2 / 2016)$ and Balcarres male and female wards (29/2/2016).

During this phase, we monitored the length of stay for consecutive discharges from the five wards to ascertain whether the introduction of SCAMPER had any impact on this. We hypothesised that improving the structure of ward rounds would potentially reduce the length of stay.

\section{RESULTS}

\section{Implementation and compliance}

The standardised ward round document, based around the acronym SCAMPER, was piloted on a paired male and female ward in November 2015. A 'Guide to SCAMPER' (see online supplementary appendix 1) was distributed to all wards to ensure standardised education for staff. Verbal information and support was also available to the wards as they introduced the form.

Its use was audited over a 15 -week period. Within 8 weeks, it was being used for $100 \%$ of patients and this was maintained over the next 7 weeks.

SCAMPER and a slightly modified SHAMPER were subsequently introduced into three other acute psychiatry wards and the IPCU respectively, between January and March 2016. There was a total of 112 beds across these wards.

Rates of completion of SCAMPER/SHAMPER were audited. Mean rates of completion across the wards varied between $62 \%$ and $95 \%$ (see figures 2-5). It was noted that at medical staff change over in April 2016 rates of completion decreased. The importance of giving new staff guidance and training on the use of the structured ward round became apparent. In May 2016, a consultant check of the SCAMPER form was also introduced to ensure adequate documentation at each ward round entry.

\section{Feedback}

An initial questionnaire was distributed to clinical staff in February 2016 on the pilot wards (Balcarres male and female wards) to assess awareness of and satisfaction with SCAMPER and suggested areas for improvement (see online supplementary appendix 2). This was completed 
Percent

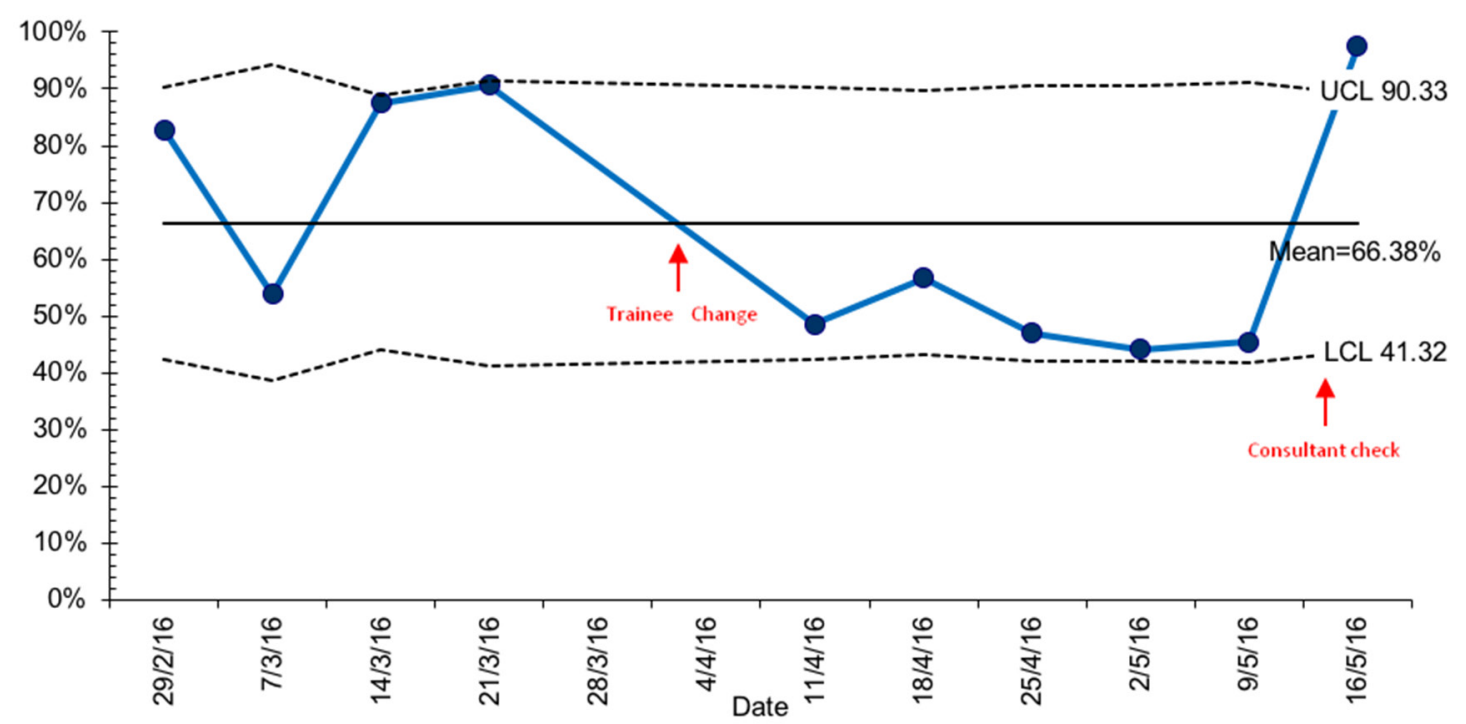

Figure 2 Rates of completion of SCAMPER on Balcarres wards. ECT, electroconvolsive therapy; IPCU, intensive psychiatric care unit; LCL, lower control limit; UCL, upper control limit.

by eight team members: three charge nurses, four staff nurses, one consultant psychiatrist. Seventy-five per cent had heard of SCAMPER and 50\% had used SCAMPER. Feedback was constructive and positive, reporting that SCAMPER made ward rounds focused, structured and concise. Participants felt the form was easy to complete and contained relevant topics for discussion within the MDT. The fact that SCAMPER had been developed locally and could be adapted to the needs of REH was positively received. Task assignment was felt to be beneficial and participants felt SCAMPER was helpful for continuity, as each week reference could be made to the previous week's SCAMPER. A suggested area of improvement was enabling SCAMPER to be completed electronically; however, this was not possible due to IT access limitations.

In May 2016, a paper questionnaire was distributed throughout all the acute psychiatry wards using SCAMPER and the IPCU (See online supplementary appendix 3). All staff were invited to participate and 41 responded. Questionnaires were completed by a range of staff including staff nurses, charge nurses, nursing assistants, occupational therapists, doctors at various grades and pharmacists.

Of the 41 respondents, $90 \%$ had heard of SCAMPER and $88 \%$ had been using SCAMPER. Results from the questionnaire were largely positive, with $78 \%$ thinking

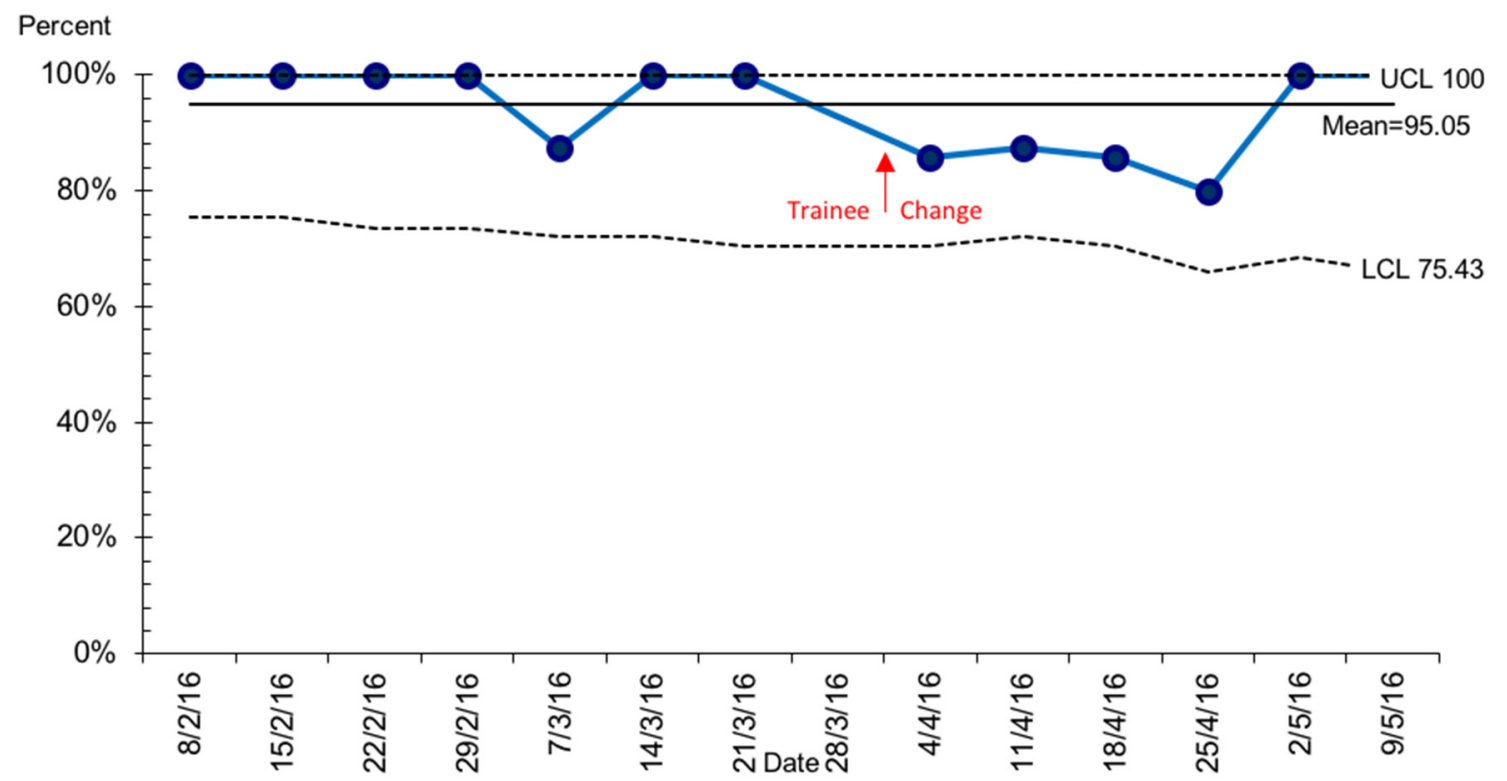

Figure 3 Rates of completion of SCAMPER on IPCU. IPCU, intensive psychiatric care unit; LCL, lower control limit; UCL, upper control limit. 


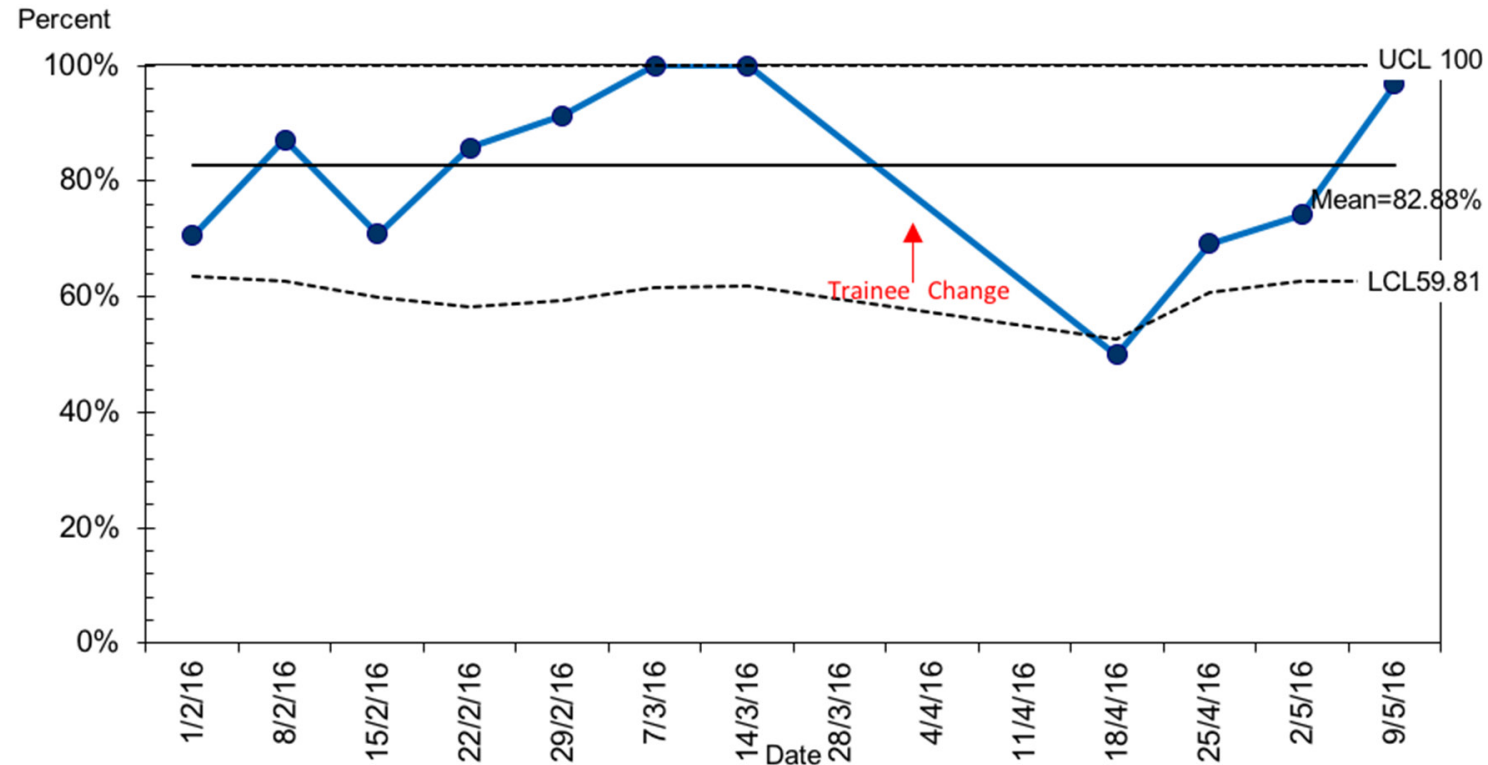

Figure 4 Rates of completion of SCAMPER on Meadows ward. LCL, lower control limit; UCL, upper control limit

that SCAMPER had improved the quality of ward rounds. Staff reported the SCAMPER ward round to be easy, clear and focused. As one respondent stated, 'It helps to be organised and clarify decision making during the ward rounds'.

The format was described as thorough and holistic. One respondent described, 'all aspects of care are reviewed weekly'. Clear standardised documentation enabled progress to be directly compared each week and there was accountability, with clear task assignment.

Some staff commented that the SCAMPER format made the ward round longer; however, $66 \%$ of respondents felt it had no impact on the length of the ward round. Seven per cent of respondents felt the ward round was actually shorter as it was more structured and focused.
The balancing measures in this project were length of preparation time, length of ward round and length of stay. Verbal feedback was that none of these were adversely affected by the introduction of SCAMPER. Indeed, one charge nurse commented that 'This appears to have made the patient flow more efficient'. Length of stay for consecutive patients at time of discharge, between October 2015 (pre-introduction of SCAMPER) and June 2016 (post-introduction of SCAMPER), was recorded (see figure 6). The results showed a median length of stay of 14 days, with normal variation. The introduction of SCAMPER had no statistically significant impact on the length of hospital stay.

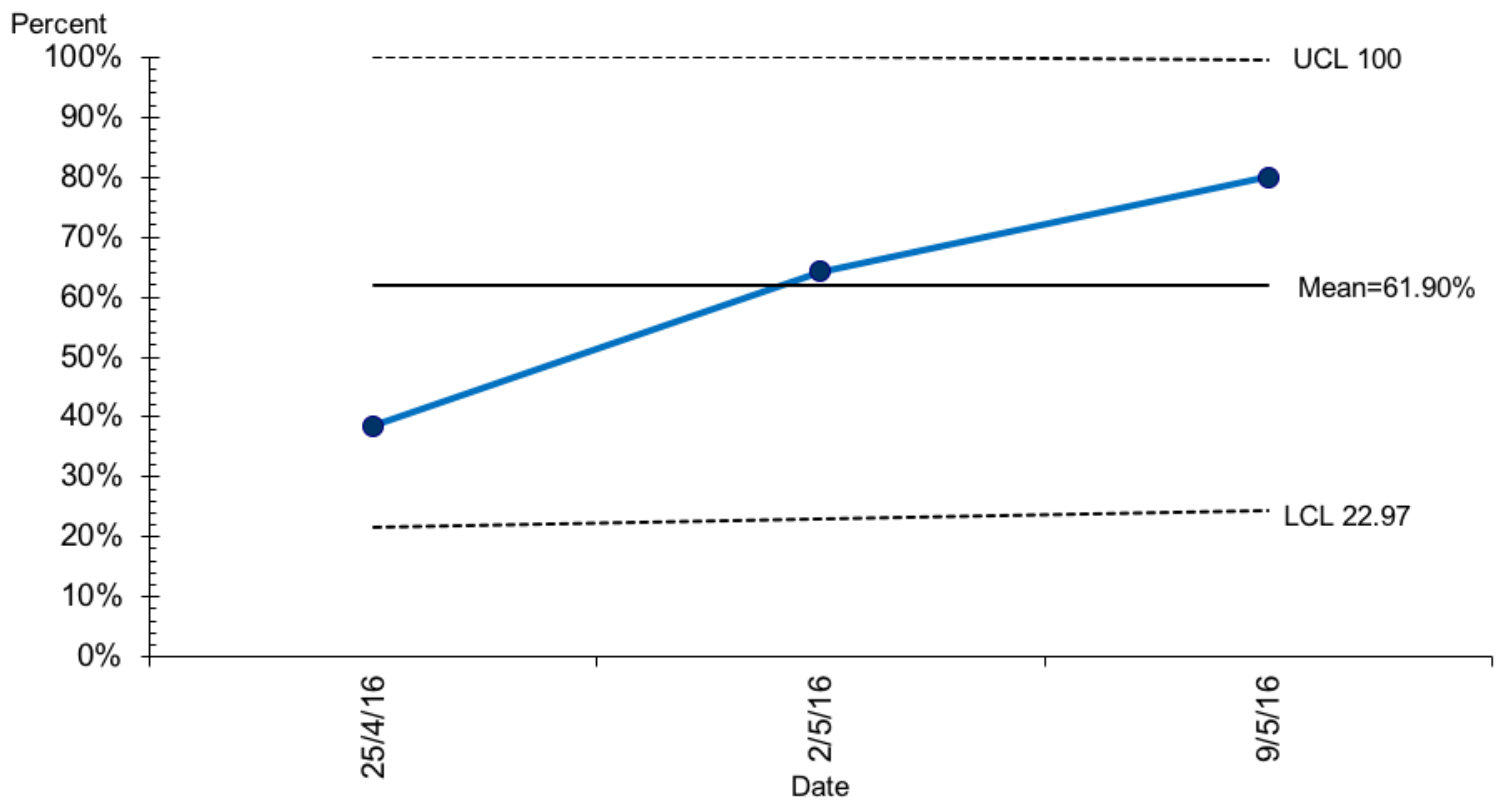

Figure 5 Rates of completion of SCAMPER on Hermitage ward. LCL, lower control limit; UCL, lower control limit. 


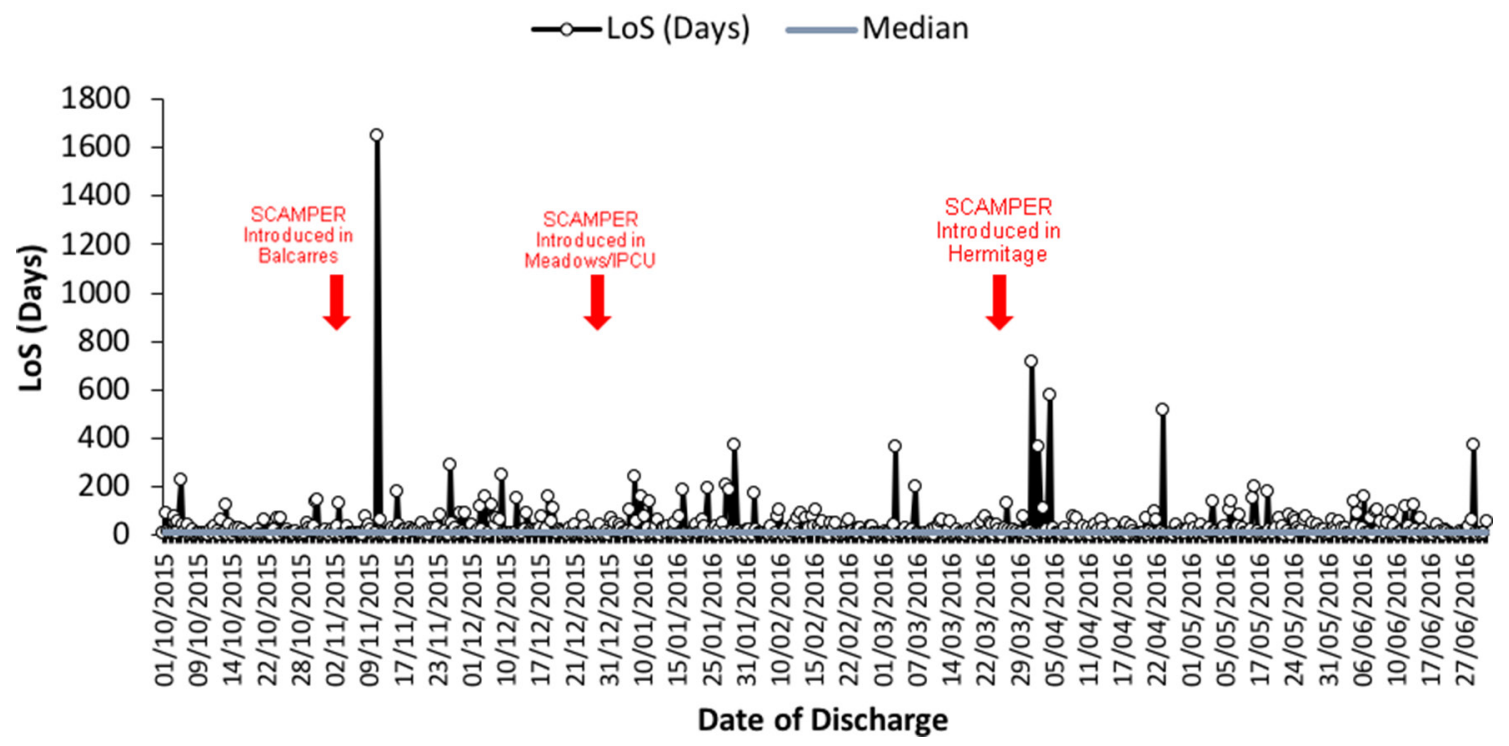

Figure 6 Length of stay in acute wards (October 2015 to June 2016). IPCU, intensive psychiatric care unit.

Suggested areas of improvement included completing the ward round electronically, having a specific section for physical health and having more structure to the nursing review section. Five per cent of respondents felt a more thorough risk assessment was needed. One respondent thought we should document who attended the ward round, while others felt more space for including patient's views was required. Respondents commented that tasks are often assigned to specific staff members but there was poor compliance with ticking off tasks when complete.

\section{Feedback from external agencies}

A planned Mental Welfare Commission visit of REH occurred in February 2016. They commented positively on the impact of SCAMPER on the standard of multidisciplinary documentation and review. 'We hope that these developments will help MDTs' documentation of risk assessments and review of risk during admission.' ${ }^{4}$ They noted positive impact on review of risk and that all detained patients had a T2/T3 and that prescribing was in line with these.

The Mental Welfare Commission also noted the positive implications of SHAMPER. 'There are sections for holistic review under a number of headings. We found that staff had completed this to a very high standard, and had documented very comprehensive weekly MDT reviews. These included documentation of physical healthcare provided and activities engaged in. This is good practice. 4

SCAMPER and SHAMPER have been well received and successfully spread throughout the hospital. There is a sense of improved team working within the wards, with a focus on quality improvement. Results have shown that structured ward round completion rates of $95 \%$ are achievable and a significant improvement on previous care practices, but that there is still work to do to improve compliance and reduce variation between wards.

\section{LESSONS LEARNT AND LIMITATIONS}

This project has demonstrated that structured ward rounds do translate from general medicine to psychiatry. The structured ward rounds have been well received by the MDTs involved in psychiatry ward rounds.

Innovations developed at REH by front line staff, and supported by management, have led to a sustainable improvement in ward rounds. Initially, this was on one paired male and female acute psychiatry ward and subsequently throughout acute adult psychiatry at REH. The results show that the standardised ward round is used, and the median completion rates range from $62 \%$ to $95 \%$ across the wards. No additional funding costs have been incurred in designing and implementing the structured ward round.

Two things that have remained difficult to evaluate in this project are the quality of the ward round entries and the time taken to complete tasks post ward round. Both require further exploration. Defining what 'quality' means in regard to the documentation may be difficult, but important, and could be the focus of further improvement work. Although limited by having only 41 respondents, feedback from the questionnaires has been positive and the overwhelming feeling from participants was that ward round quality had improved. The time to complete tasks is important but is difficult to capture. This could involve additional data collection which may actually reduce efficiency.

The quality of the ward round discussion and entry should be the same for patients who are boarded to other wards. This was not evaluated and we feel there is work to be done to ensure ward round quality for boarding patients.

In order to promote patient-centred care, further study is required to determine the best way to involve patients' views in the ward round discussion. Some consultants invite patients into their ward rounds, whereas other patients do not attend. 
Initial results suggest that median length of stay has not changed significantly with the implementation of SCAMPER. As the use of the structured ward round becomes more embedded in clinical practice, further work is required to evaluate whether the structured ward rounds have impacted patient flow.

We found that at times of medical staffing change over full compliance with ward round documentation decreased. Making new staff aware of the standardised ward round documentation and providing education on completion has been addressed by the development of a PowerPoint presentation delivered by a consultant psychiatrist for induction of new medical staff. A consultant check, to ensure adequate completion of the ward round documentation during the ward round, has also been introduced.

It is still early to determine if the new ward round process is sustainable. We will address this by conducting quarterly audits of completion rates and sharing the results with ward teams so that they can monitor their performance. However, sustainability of this innovation (and hence improvement in provision of safer care) is promising given that there is now good evidence accumulating that it is 'normalised' as part of routine clinical practice in the wards participating in this study. We know from the evidence base that successful implementation and sustainability of safety and improvement interventions is largely predicated on summarising and simplifying for frontline staff what is to be done; measuring and providing feedback on performance and outcomes and improving culture by 'building expectations of performance standards into work processes'. ${ }^{1}$ Our study is addressing all of these issues and in particular that the combination of a technical solution (SCAMPER) and attitudinal expectations (that this solution is a necessary partly of routine clinical work) is making a difference to patient care practices and safety. ${ }^{5}$ We have noted the drop in completion rates with staff change over and have developed induction training for new medical staff as well as providing written information on all wards. New nursing staff are given local induction on ward round preparation and completion of the SCAMPER forms.

It is disappointing that this intervention did not produce any reduction in length of stay for patients in the wards. This is perhaps not surprising in this context. The median length of stay during this period was 13 days which is already lower than the national figure for General Adult Psychiatry which is 14 days. ${ }^{6}$ Length of stay is dependent on a range of variables such as access to outpatient care and social factors such housing and support services. These are factors which are independent of inpatient processes and cause significant delays for patients leaving hospital. Further improvement work in partnership with social work, housing and providers of community care may be beneficial, but there are also financial constraints which may limit progress in this regard.

In terms of spread of this innovation, a general adult ward in a neighbouring trust has begun to use the
SCAMPER form for ward rounds. SCAMPER has also been adapted for use in Old Age Psychiatry and subsequently introduced into a ward in the REH and a ward in another Scottish hospital. The local rehabilitation service has also expressed an interest in using the same ward round documentation. More widely, our development and implementation of a structured ward round for acute adult psychiatry should be of interest to psychiatrists and their clinical teams around the UK, and internationally, who also have a paucity of a structured, holistic ward round documentation system.

\section{CONCLUSION}

Adopting a participatory, codesign process with experienced and knowledgeable frontline staff has led to the innovative development and successful implementation of a standardised ward round system to address identified quality care issues. Compliance with the standardised ward round structure has been high and the questionnaire highlights that staff feel ward round quality and documentation has improved. Completion rates of $95 \%$ are achievable but there is still work to do to improve compliance and reduce variation in completion between wards. Ongoing PDSA cycles will continue to be applied to monitor and improve the structured ward round and compliance with it. Individual improvement projects to lead to improvement in the outcomes of the seven domains of SCAMPER will be conducted. For example, a project to ensure assigned tasks are completed and signed off could be conducted.

A structured and holistic standardised ward round has been well received by the MDT. Steps have been taken to improve the standardised document during the process based on feedback and PDSA cycles. Questionnaire responses found ward rounds to be more focused, organised and holistic. Anecdotally staff report earlier interventions, anticipation of issues that may delay discharge, and more thorough risk assessment. With this feedback in mind, this project has arguably had a positive impact on patient safety and care. The balancing measures in this project were length of preparation time, length of ward round and length of stay. None of these were adversely affected by the introduction of SCAMPER.

Structured ward rounds are not widely used within adult psychiatry and have not previously been used at the REH. Other psychiatry departments locally and in neighbouring trusts have expressed interest in using this structured ward round format and documentation.

Acknowledgements We would like to thank all the staff at the Royal Edinburgh Hospital who have been involved in the development, implementation and improvement of the SCAMPER and SHAMPER ward round documentation. We would like to thank medical ward staff who helped with data collection. We would like to thank Mr Paul Bowie from NHS Education for Scotland for his guidance on writing this report and Katie Steel from Lothian Analytical Services for help creating the graphs.

Contributors ARM assisted in the development of the project and data collection, performed analysis of results and wrote the manuscript. SJC initiated the project, completed the tests of change, performed data collection, performed analysis of 
results and supervised writing of the manuscript. She will act as corresponding author as is responsible for ongoing development of the project.

Funding The authors have not declared a specific grant for this research from any funding agency in the public, commercial or not-for-profit sectors.

Competing interests None declared.

Patient consent Not required.

Provenance and peer review Not commissioned; externally peer reviewed.

Open access This is an open access article distributed in accordance with the Creative Commons Attribution Non Commercial (CC BY-NC 4.0) license, which permits others to distribute, remix, adapt, build upon this work non-commercially, and license their derivative works on different terms, provided the original work is properly cited, appropriate credit is given, any changes made indicated, and the use is non-commercial. See: http://creativecommons.org/licenses/by-nc/4.0/.

\section{REFERENCES}

1. Mental Health Commission. Multidisciplinary team working: from theory to practice. 2006.

2. Cresswell J, Beavon M. Accreditation for Inpatient Mental Health Services. In: Standards for inpatient wards - working-age adults. 4th edn: Royal College of Psychiatrists, 2010.

3. Royal College of Physicians and Royal College of Nursing. Ward rounds in medicine: principals for best practice. London: RCP, 2012.

4. Mental Welfare Commission for Scotland. Report on announced visit to: Royal Edinburgh Hospital, Meadows Male, Meadows Female, Balcarres Male, Balcarres Female. www.mwcscot.org.uk/media/ 316739/royal_edinburgh_hospital_meadows_and_balcarres_wards_ 4_february_2016.pdf (accessed 4 Feb 2016).

5. Bosk CL, Dixon-Woods M, Goeschel CA, et al. Reality check for checklists. Lancet 2009;374:444-5.

6. ISD Scotland National Benchmarking Project. Mental Health Benchmarking Toolkit 2015/2016. www.isdscotland.org/Health-Topics/ Quality-Indicators/Publications/data-tables2017.asp?id=2010\#2010 